\title{
Regulator of sex-limitation (Rsl) encodes a pair of KRAB zinc-finger genes that control sexually dimorphic liver gene expression
}

\author{
Christopher J. Krebs, ${ }^{1}$ Leslie K. Larkins, ${ }_{1}^{1}$ Ryan Price, ${ }^{1}$ Kathryn M. Tullis, ${ }^{1}$ Raymond D. Miller, ${ }^{2}$ and \\ Diane M. Robins ${ }^{1,3}$ \\ ${ }^{1}$ Department of Human Genetics, University of Michigan Medical School, Ann Arbor, Michigan 48109-0618, USA; \\ ${ }^{2}$ Division of Dermatology, Washington University, St. Louis, Missouri 63110, USA
}

Sexually dimorphic expression of a broad array of liver proteins involved in reproduction and xenobiotic metabolism is induced at puberty by sex-specific growth hormone patterns. An additional control of sex-dependent gene expression is conferred by Regulator of sex-limitation (RsI) alleles. In variant rsl mice, females inappropriately express the male Sex-limited protein, Slp. We recently showed that a panel of male-specific liver genes is repressed by $R s l$, accentuating sex differences in a hormone-independent manner. Here we map rsl to a region on Chromosome 13 comprised exclusively of KRAB (Kruppel-associated box) zinc-finger protein (ZFP) genes. Among eight Rsl candidate (Rslcan) genes within the critical genetic interval, the recent duplicates Rslcan-4 and Rslcan-9 both harbor mutations in rsl mice (partial deletion and splice-site inactivation, respectively). Transgenesis with bacterial artificial chromosome (BAC) clones encompassing Rslcan-4 restores male-specific MUP (major urinary protein) expression to rsl mice, whereas a BAC containing Rslcan-9 rescues sex-specific expression of Slp and cytochrome P450 Cyp2d9. Thus, the Rslcan-4 and Rslcan-9 paralogs partitioned regulation of their target genes during evolution. This demonstrates the first biological role for a set of KRAB zinc-finger repressor proteins and reveals the molecular basis of a gene-silencing pathway critical for sexual dimorphism.

[Keywords: Regulator of sex-limitation; sex-limited protein (Slp); KRAB zinc-finger protein; sexual dimorphism; liver gene expression]

Received July 23, 2003; revised version accepted September 2, 2003.

Physiological differences between males and females exist in a variety of tissues, including some not usually considered sexually dimorphic. The rodent liver is one example that has provided an excellent model for investigating the complex interplay of hormonal, developmental, and tissue-specific control of gene expression (Gustafsson et al. 1983). Sex-specific patterns of liver gene expression occur for several cytochrome P450 enzymes involved in steroid and xenobiotic metabolism, as well as for certain proteins that function in reproductive physiology and behavior (Roy and Chatterjee 1983). Hepatic sex differences begin at puberty, when gonadal steroids initiate pituitary secretion of growth hormone $(\mathrm{GH})$ in sex-specific patterns. The male GH pulse profile has high amplitude peaks and low troughs, whereas fe-

${ }^{3}$ Corresponding author.

E-MAIL drobins@umich.edu; FAX (734) 763-3784.

Article published online ahead of print. Article and publication date are at http://www.genesdev.org/cgi/doi/10.1101/gad.1135703. male pulses are more frequent and less extreme, resulting in more stable levels of GH in serum (Lund et al. 1991; Mode et al. 1992). Pulsatile GH effects are transmitted by the signal transducer and activator of transcription (STAT) factor 5b (Waxman et al. 1995; Gebert et al. 1997). The extent and duration of STAT5b activation is dependent on the nature of GH signaling, as well as interactions with numerous other nuclear and cytoplasmic proteins (Waxman 2000). Similar GH regulation occurs in humans, where hepatic sex differences may prove relevant to pathophysiologies that vary with gender, such as differences in drug metabolism and incidence of certain diseases, as well as problems related to pregnancy (van den Berg et al. 1996; Veldhuis 1996; Jaffe et al. 1998).

A fascinating clue to the complexity of sexually dimorphic liver gene expression is unmasked by the mouse Rsl (regulator of sex-limitation) locus, first described as a modifier of sex-limited protein (Slp) gene expression (Brown and Shreffler 1980). At puberty, female mice 
KRAB-ZFPs confer sex-limited expression

from inbred strains with rsl alleles express SIp, a diverged complement component $\mathrm{C} 4$ gene, which is normally expressed only in adult male liver and kidney (Shreffler 1982). Moreover, males of these strains also have elevated SIp levels (Jiang et al. 1996). SIp expression in Rsl mice is regulated by androgen either directly in the kidney, or indirectly in the liver through its effect on pulsatile GH release by the pituitary (Georgatsou et al. 1993; Nelson and Robins 1997). The variation in S1p expression seen in rsl mice suggests that in addition to hormonal induction, gene repression is required for sexspecific expression patterns. Using congenic strains carrying rsl alleles from PL/J and FM mice (B10.D2.PL, B10.D2.FM), we recently characterized Rsl action in greater detail (Tullis et al. 2003). Rsl affects SIp in liver, but not in kidney, and acts on a broad array of malespecific liver genes. These include major urinary proteins (MUPs) that function as pheromone carriers (Bacchini et al. 1992) and cytochrome P450 Cyp2d9 (16 $\alpha$ hydroxylase), which is expressed predominantly in males and metabolizes androgens (Lakso et al. 1991). Genes expressed in female-prominent patterns, such as Cyp2a4, are not affected by Rsl. Because rsl alleles are recessive and cause increased expression of their target genes, the Rsl gene product most likely functions in transcriptional repression. This repression accentuates the magnitude of the hormonally induced male-female difference, thus enforcing sex-limited expression.

The hypothesis that Rsl dictates transcriptional repression is borne out by the positional cloning presented here. Genetic and physical mapping pinpointed a contiguous cluster of $>20 \mathrm{KRAB}$ zinc-finger protein (ZFP) genes as candidates for Rsl. The largest class of genes in the human genome, and second largest in the mouse after odorant and pheromone receptor genes, are those encoding proteins with Kruppel-type $\mathrm{Cys}_{2} \mathrm{His}_{2}$ zinc-finger DNA-binding domains (Mouse Genome Sequencing Consortium 2002). The largest subfamily of ZFPs, numbering several hundred genes, contains an additional domain called the Kruppel-associated box (KRAB), which functions in vitro as a powerful repressor (Margolin et al. 1994; Mark et al. 1999; Thiel et al. 2001). KRAB-ZFP genes are abundant and broadly dispersed in mammalian genomes, often in tandem arrays suggestive of multiple duplication events (Tang et al. 2002). Their importance is inferred from their recent origin and subsequent rapid expansion in vertebrate lineages, but their role in vivo is unclear because biological functions have not been identified (Looman et al. 2002; Tanaka et al. 2002). We show that two of these KRAB-ZFP genes, whose high degree of identity implies their recent duplication, have sequence variations accounting for rsl phenotypes, and in transgenic $r s 1$ mice, each restored appropriate expression of a distinct subset of the male-specific liver genes. Thus, this pair of KRAB zinc-finger genes acts together to perform the function of Rsl. Our identification of Rsl sheds light on the molecular mechanism of sexually dimorphic gene expression, and moreover, provides an entrée into mechanistic studies of KRAB-ZFP action by this first assignment of a biological function.

\section{Results}

Variation of the Rsl phenotype in inbred mouse strains

Allelic variation existing in inbred strains of mice enhanced our ability to clone Rsl. Congenic B10.D2, B10.D2.PL, and B10.D2.FM mice (Jiang et al. 1996) exemplify variation at the Rsl locus because the genetic backgrounds are essentially identical (Fig. 1). Functional differences in the recessive $r s l^{P L}$ and $r s l^{F M}$ alleles, derived from PL/J and FM strains, respectively, are demonstrated by differential effects on their target genes (Tullis et al. 2003). For example, Slp mRNA levels are much higher in $r s l^{P L}$ than $r s l^{F M}$ females, as determined by RNase protection assays (Fig. 1A). To simplify cloning, we expanded analysis of Rsl target genes to include the common inbred strains C57BL/6J (B6) and 129/SvEvTac (129). 129 males have significantly lower Slp levels than B10.D2 males because of cis-acting regulatory differences in the SIp gene itself, but female expression is substantial, that is, qualitatively similar to the rsl strains B10.D2.PL and B10.D2.FM. B6 mice have a null Slp allele, but show pronounced sex-limited MUP expression (Fig. 1B), indicating that they are similar to the B10.D2

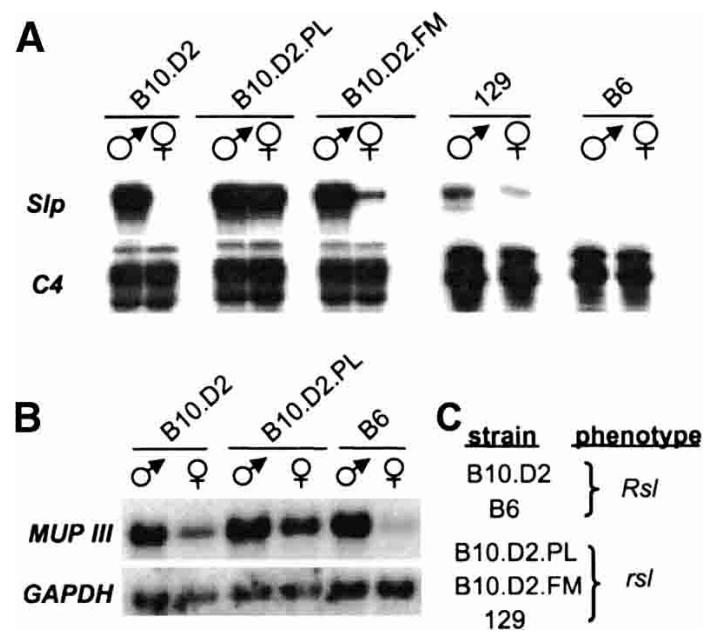

Figure 1. Rsl variation revealed by differential effects on malespecific target genes. $(A)$ Total liver RNA from congenic and inbred mice was assayed for $S 1 p$ by RNase protection (RPA), using a probe that also detects Slp's homolog C4 (Cox and Robins 1988). Elevated Slp levels in B10.D2.PL, B10.D2.FM, and 129 , relative to B10.D2, females are hallmarks of the rsl phenotype. rsl allele-specific differences are evident in lower SIp levels for $r s I^{F M}$ than $r s l^{P L}$ mice. Allelic variation also exists in target genes; for example, B6 mice do not express Slp. C4 is an internal control that is not sex-dependent. (B) Mouse urinary proteins (MUPs) were analyzed by Northern blot of liver RNAs using an oligonucleotide probe complementary to MUP III (Shahan et al. 1987), which is elevated in both sexes of $r s 1^{P L}$ mice (more obvious in females because of their normally low expression), relative to B10.D2 mice. B6 mice are Rsl based on dimorphism for MUP III. The filter was reprobed with GAPDH cDNA as a loading control. $(C)$ Summary of phenotypes. Strains with strongly dimorphic expression of male-specific liver genes are considered wild type for Rsl; rsl strains are those in which females have lost regulation of sex-limitation of liver target genes. 
strain in Rsl phenotype. Classification of the B6 and 129 strain phenotypes as Rsl versus rsl, respectively (Fig. 1C), is in accord with DNA typing using polymorphic microsatellite markers that indicated B10.D2 mice were indistinguishable from B6, and B10.D2.PL mice were indistinguishable from 129, within the Rsl locus. This allowed us to use existing genomic libraries from the common strains for cloning.

Fine-scale genetic and physical mapping of the Rsl region

Using an intercross strategy $[(\mathrm{PL} / \mathrm{J} \times \mathrm{B} 10 . \mathrm{D} 2) \times(\mathrm{PL} /$ $\mathrm{J} \times \mathrm{B} 10 . \mathrm{D} 2)]$, we narrowed the $R s 1$ genetic interval, previously mapped to a $1.2-\mathrm{cM}$ region of mouse Chromosome 13 (Jiang et al. 1996). Then 1660 F2 mice were genotyped with polymorphic microsatellite markers flanking Rsl, and recombinants were revealed by their homozygosity for one marker and heterozygosity for the other (Fig. 2). The Rsl allelic status could be directly determined for F2 females when the homozygous flanking marker was $\mathrm{PL} / \mathrm{J}$, because the presence of liver Slp mRNA in females denotes homozygosity for rsl. For recombinants in which the homozygous flanking marker was B10.D2 and for males, because their quantitative Slp differences are not robust enough for scoring, the RsI genotype was determined by testing female progeny from backcrosses to PL/J mice. Such analysis of the 15 recombinants with breakpoints between D13Mit13 and D13Mit281 narrowed the nonrecombinant interval to $0.2 \mathrm{cM}$ (Fig. 2).

A unique DNA probe flanking the linked marker D13Mit66 was used to screen a C57BL/6J genomic BAC library (Osoegawa et al. 2000). Complete or partial clone sequences in the National Center for Biotechnology Information (NCBI) High-Throughput Genome Sequence (HTGS) database allowed assembly of a contig of six BACs with a minimum tiling path spanning $\sim 1 \mathrm{Mb}$ (Fig. 3A). Linkage analysis with polymorphic markers derived from these BAC sequences (D13Dmr markers) refined the critical genetic interval to $\sim 200 \mathrm{kB}$.

Candidate Rsl genes (Rslcans) were found by comparing BAC sequences to nonredundant and mouse EST databases (Burge and Karlin 1997). The first gene identified, on BAC 163-B-15, was myeloid zinc finger 22 (MZF22), which has highly conserved KRAB A and B domains (Fig. 3B,D; Mark et al. 1999). These domains first appeared in vertebrate lineages and, coupled with Kruppel-like zinc fingers, specify one of the largest subfamilies of ZFPs (Ravasi et al. 2003). In vitro evidence suggests the proteins act as monomeric transcriptional repressors, but in vivo functions are largely unknown (Margolin et al. 1994; Thiel et al. 2001; Looman et al. 2002). This capacity for negative regulation makes them compelling candidates for Rsl. We identified 22 additional KRAB-ZFPs in the BAC contig and named them Rslcans in their order of discovery. The only other gene within this region was a processed pseudogene similar to acidic ribosomal protein P0 $(A r b p)$. Eight of the Rslcans lay within the critical genetic interval (Fig. 3C). Their intriguing pair-

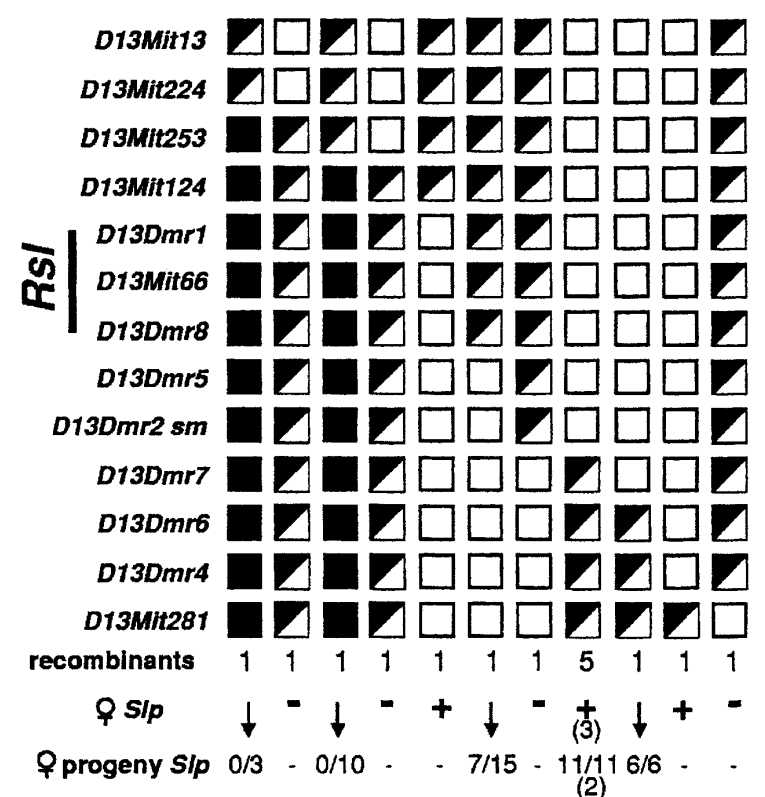

Figure 2. High-resolution genetic map of the Rsl locus. Using a PL/J $\times$ B10.D2 intercross, $1660 \mathrm{~F} 2$ mice were typed with polymorphic microsatellite markers (listed at left). D13Mit markers were from Research Genetics; D13Dmr markers were developed from the B6 BAC contig (see Fig. 3). Open boxes indicate homozygosity for the PL/J allele, filled boxes homozygosity for the B10.D2 allele, and diagonally half-filled boxes indicate heterozygosity. At the bottom is the number of recombinants that share the haplotype indicated. Scoring for liver Slp expression in directly informative females (those having a homozygous PL/J flanking marker) is shown on the line below (o Slp), indicated as positive $(+)$ or negative $(-)$. Within this group, the recombinant female with a breakpoint between D13Mit124 and D13Dmr1 defined the centromeric (top) side of the Rsl interval. For all other recombinants, testing of female backcross progeny is shown below the arrows as the proportion positive for Slp. The telomeric side of the Rsl interval, between D13Dmr8 and D13Dmr5, was defined by the single recombinant with 7 of 15 female progeny positive for Slp.

wise similarity suggests that a region encompassing four genes underwent duplication and subsequent inversion(s): Rslcan-17 is most similar to Rslcan-14, Rslcan-9 to Rslcan-4, Rslcan-10 to Rslcan-13, and Rslcan-7 to Rslcan-6. For example, Rslcan-4 differs from Rslcan-9 by only one amino acid in the highly conserved KRAB A domain, and Rslcan-14 and Rslcan-17 differ from each other in this region by only two residues, but they differ from Rslcan-4 and Rslcan-9 by five. In the less conserved KRAB B domain, Rslcan-4 and Rslcan-9 differ from each other by three amino acids, but each differs from Rslcan14 or Rslcan-17 by at least 12 amino acids (nearly $40 \%$ ). The duplications and inversions that have diversified this locus are common to numerous clusters of KRABZFP genes in mouse and human genomes, and may underlie rapid evolution of proteins with related but distinct functions (Shannon et al. 1998; Looman et al. 2002; Tanaka et al. 2002).

A generic KRAB-ZFP gene structure is shown in Figure 
A

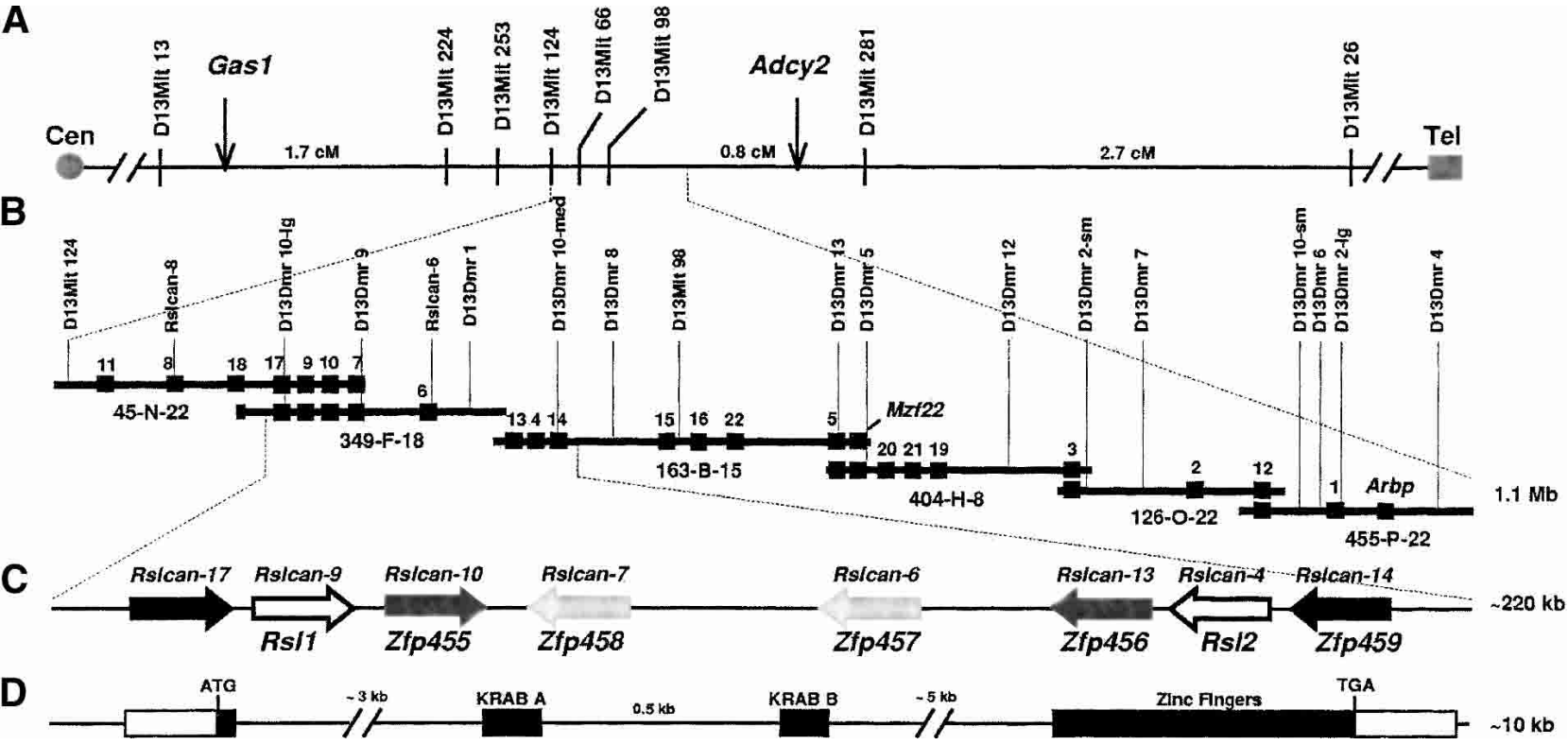

Figure 3. Physical mapping of the Rsl locus. (A) A genetic map was derived by genotyping eight MIT microsatellite markers in 15 recombinant mice. The recombination frequencies are shown in centimorgans between markers. The previously mapped growth arrest specific gene 1 (gas1) and adenylate cyclase 2 (adcy2) are indicated. (B) Six BACs (solid lines, with address names below) form a contig spanning the Rsl locus. The 16 BAC-derived microsatellite markers and two polymorphic Rsl candidate genes (Rslcan-8 and Rslcan-6) used in mapping are indicated above the contig. Black boxes indicate Rsl candidate KRAB zinc-finger genes, numbered in the order of their discovery. Mzf22 is the only previously reported gene in this region, along with Arbp, a processed pseudogene. (C) Schematic of eight candidate genes within the critical genetic interval. Shade or pattern indicates their pairwise similarity. Rslcan-17 is most similar to Rslcan-14, Rslcan-9 to Rslcan-4, Rslcan-10 to Rslcan-13, and Rslcan-7 to Rslcan-6. The Human Genome Nomenclature Committee has assigned names to these genes (Zfps455-460 and Rsl1 and Rsl2) in accord with functional identification of the latter two genes in this report. Arrowheads indicate the direction of transcription. (D) Genomic architecture of a prototypical KRAB zinc-finger gene from the Rsl locus. Black boxes represent the coding regions within the exons, with approximate distances (in kilobases) between exons given. ATG and TGA indicate start and stop codons, respectively. Exon 1 typically encodes a short ( 2-12-amino acid) N-terminal leader, prior to the highly conserved KRAB A domain, which starts immediately within exon 2. KRAB A and B domains encompass repressor activity, tethered to DNA via a variable number of zinc fingers located in the fourth and last exon.

3D, for comparison to the Rslcan genes. The transcriptional repressor function resides in the 42-amino acid KRAB A and 32-amino acid KRAB B domains, which are encoded in separate exons. In all of the Rslcan genes, these exons are separated by a small $(\sim 0.5 \mathrm{~kb})$, highly conserved intron. The KRAB domains repress transcription by recruiting the universal corepressor, KAP-1, which acts as a scaffold for chromatin-condensing proteins (Friedman et al. 1996; Schultz et al. 2001). Variation in the sequence of the KRAB domains might influence efficiency of interaction with components of a repression complex. The DNA-binding $\mathrm{C}_{2} \mathrm{H}_{2}$ zinc fingers, ranging in number from 6 in Rslcan-14 to 23 in Rslcan-7, are encoded in the fourth and final exon. The apparent paralogs, Rslcan-4 and Rslcan-9, for example, each have 14 finger motifs, with an overall amino acid sequence similarity of $94 \%$. Of these motifs, some vary at a critical Cys or His that would likely prevent finger formation, and we thus term them "pseudofingers." Rslcan-9 has two pseudofingers (the first and sixth); Rslcan-4 is the same, and additionally its second and seventh fingers are degenerate. Variation in zinc-finger number and sequence has the potential to alter target gene recognition and/or affinity (Gebelein and Urrutia 2001) and thus regulatory function.
Previously mapped genes that flank rsl on Chromosome 13 are growth arrest specific gene 1 (gas1) and adenylate cyclase 2 (adcy2; Fig. 3). The human orthologs of gas1 and adcy2 are located on Chromosomes 9 and 5, respectively, indicating that the $R s l$ phenotype maps to a break in conserved synteny. Clusters of zinc-finger genes have been noted at other breaks in synteny, suggesting the rapid evolution of these genes may have been subsequent to the man-mouse divergence (Dehal et al. 2001; Smith et al. 2002). This increases the difficulty of identifying a human Rsl ortholog by sequence homology and/ or genomic position.

Correlation of sequence variations in Rslcan-4 and Rslcan-9 with the rsl phenotypes

To determine which candidate within the genetically defined interval is responsible for the rsl phenotype, we sought DNA sequence and RNA expression differences that would account for functional allelic variation. This included extensive sequencing of both genomic DNA and reverse transcriptase PCR (RT-PCR) products. Genespecific analysis was confounded by the extensive homology between these KRAB-ZFPs and the hundreds of others in the genome. One successful approach to over- 
Krebs et al.

come this difficulty was to PCR-amplify segments of multiple related genes that were then resolved by genespecific restriction digestion.

Combining this strategy with Southern blots of genomic DNA revealed that Rslcan-4 and Rslcan-9 were consistently polymorphic in rsl strains, unlike the other three gene pairs in the interval. Figure 4 shows this type of analysis for a PstI polymorphism, similar to additional polymorphisms found with HindIII and MfeI. PstI cleaves a 1.4-kb PCR fragment of the Rslcan-9 zinc-finger domain into two smaller fragments but does not cleave Rslcan-4 (Fig. 4A,C). Using this diagnostic digest, we determined that wild-type mice (B10.D2, B6) contain both Rslcan-9 and Rslcan-4 genes, which are found separately in BAC clones 163-B-15 (Rslcan-4) and 349-F-18 (Rslcan-9). In contrast, rsl mice (B10.D2.PL, 129) lack the Rslcan-4 fragment. Southern blots with genomic and BAC DNAs, using a probe hybridizing to Rslcan-4 and Rslcan-9 and an adjacent gene, Rslcan-13 (Fig. 4B,D), corroborate the absence of fragments specific to Rslcan-4 in rsl DNAs. Together these results suggest Rslcan-4 is deleted in rsl mice. Comparison of sequences between strains, however, revealed that the deletion is not simply the excision of DNA, but rather is caused by homologymediated recombination between neighboring genes, resulting in a hybrid sequence with the 5' flank of Rslcan-4
A
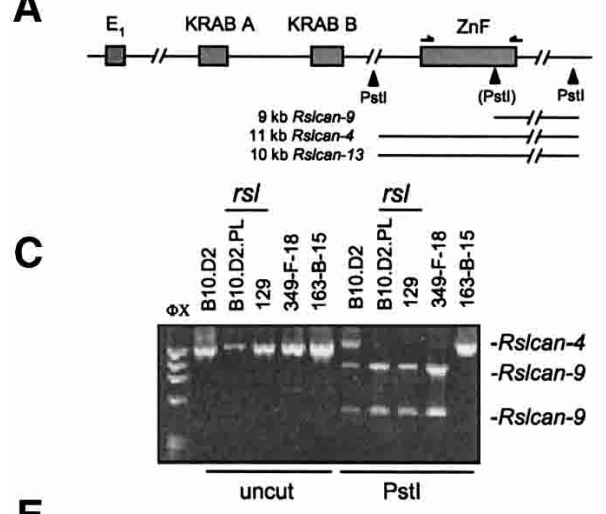

E
B

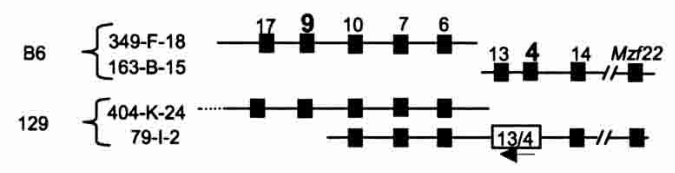

D

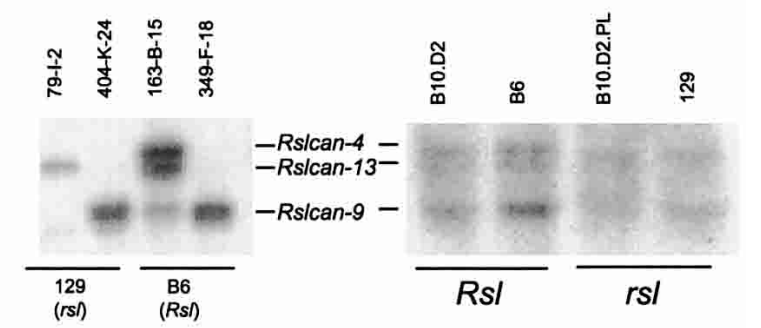

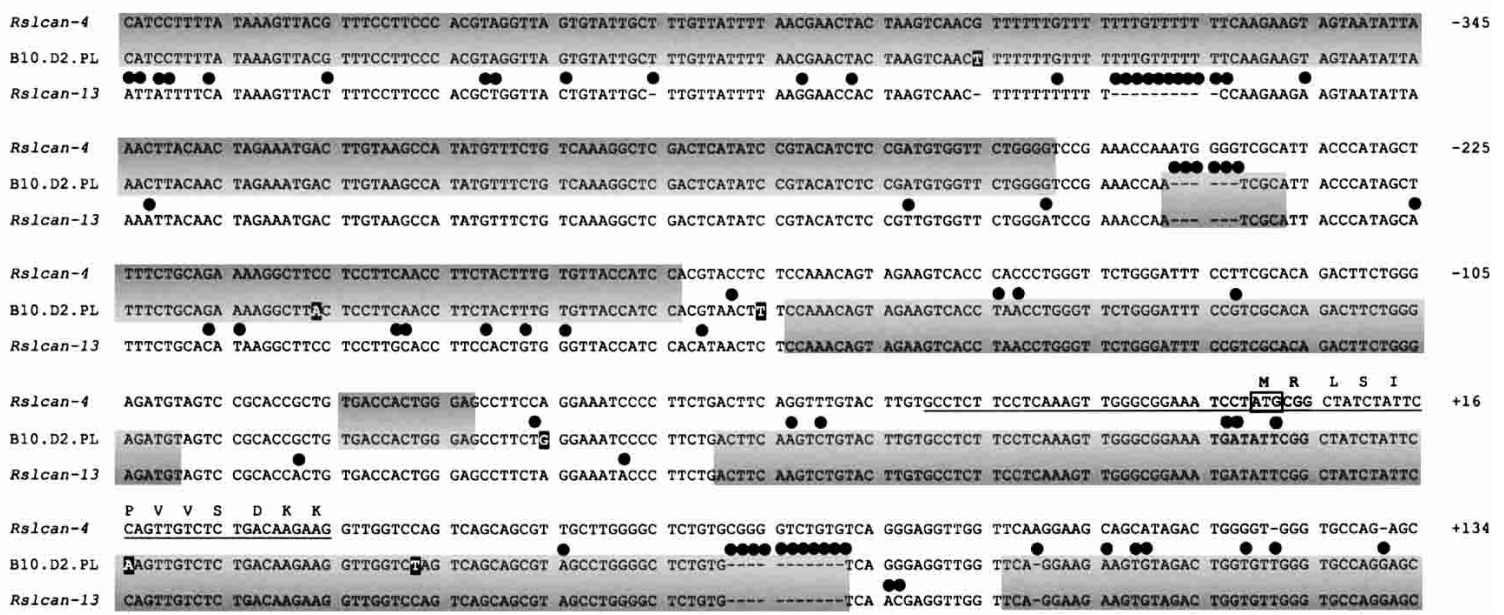

Figure 4. Deletion of Rslcan-4 sequences by recombination with Rslcan-13 in rsl mice. $(A)$ The common structure of Rslcan genes is shown, with PCR primers flanking the zinc fingers and a PstI site unique to Rslcan-9 in parentheses. Below are the genomic PstI fragments revealed by a probe that cross-hybridizes with Rslcan-4, Rslcan-9, and the adjacent Rslcan-13. (B) Map of overlapping BACs from B6 and 129 libraries (BAC addresses are on the left). Numbered black boxes indicate Rslcan genes determined by PCR. An open box and arrow indicate the Rslcan-4/Rslcan-13 hybrid discovered by sequencing. $(C)$ PCR products from zinc-finger regions of $R$ slcan-4 and Rslcan-9 were digested with Pst $\mathbf{I}$; Rslcan-4 remains undigested $(\sim 1.4 \mathrm{~kb})$, while Rslcan-9 is cleaved into bands of 942 and $450 \mathrm{bp}$. PCR products were analyzed from B10.D2, B10.D2.PL, and 129 mouse DNAs and BACs 349-F-18 and 163-B-15. The absence of Rslcan-4 bands from B10.D2.PL and 129 mice suggests the gene is deleted in rsl strains. $(D)$ Southern blot of BAC (left) and mouse genomic (right) DNAs hybridized with an Rslcan-4 zinc-finger probe shows two large bands in BAC 163-B-15 and in genomic DNAs from B10.D2 and B6 mice, representing Rslcan-4 and Rslcan-13 fragments. A single intermediate band in BAC 79-I-2 and genomic DNAs from B10.D2.PL and 129 mice corroborates fusion of the genes to an Rslcan-4/Rslcan-13 hybrid in rsl mice. (E) In aligned sequences of Rslcan-4 and Rslcan-13 with B10.D2.PL genomic DNA, shading indicates regions of alternating identity between B10.D2.PL and Rslcan-4 or Rslcan-13. The start codon (open box) and amino acids encoded in exon 1 of Rslcan-4 are shown. Filled circles highlight single-base differences; black boxes are bases unique to B10.D2.PL. The alternating regions of identity extend for an additional $10 \mathrm{~kb}$, resolving into complete identity with Rslcan-13 near the 5'-end of the zinc-finger domain in exon 4 . Genomic sequence from B10.D2.PL DNA is identical to 129 in this region, even for the B10.D2.PL-specific bases. 
fused to the coding region of Rslcan-13 (Fig. 4E). In fact, multiple putative exchanges were detected by regions of sequence identity with Rslcan-4 or Rslcan-13 (revealed by single nucleotide polymorphisms) that intermingle for $\sim 10 \mathrm{~kb}$ until finally resolving within exon 4 to complete identity with Rslcan-13. A similar phenomenon noted in hybrid LINE1 elements is referred to as "toggling" (Symer et al. 2002). The apparent deletion of Rslcan-4 in rsl mice is thus the result of toggling between sequences of the previously adjacent Rslcan-4 and Rslcan-13.

The sequence of Rslcan-9, the paralog of Rslcan-4, also revealed a mutation correlating with the rsl phenotype. The fifth base in the splice donor sequence of the first intron (IVS + 5) is T in rsl strains but G in wild-type mice and in the consensus splice donor sequence (Fig. 5A). Mutation of this base in other genes abrogates splicing by disrupting precursor mRNA-U1 snRNA pairing (Wassarman and Steitz 1992; Danckwardt et al. 2002). Indeed, Rslcan-9 splicing is altered in rsl mice (Fig. 5B). Rslcan-9 exon 1 has an AUG context reasonably fitting the translation start consensus (Kozak 2002), followed by two possible splice sites. A transcript using the first splice

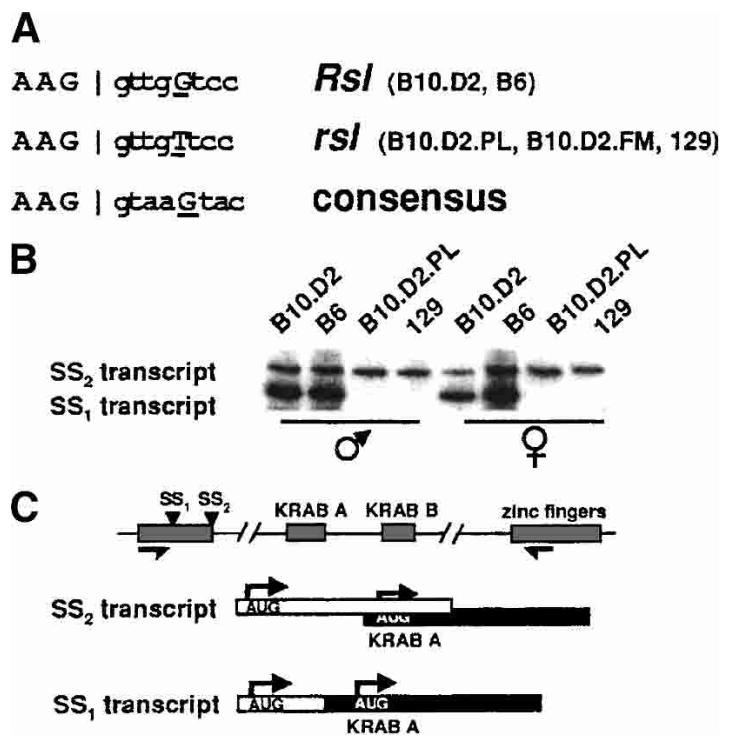

Figure 5. Identification of a splice-site mutation in Rslcan-9. (A) The $\mathrm{SS}_{1}$ splice donor sequence of the first intron of Rslcan-9 has a $\mathrm{T}$ at position +5 (IVS +5 ) in rsl strains B10.D2.PL, B10.D2.FM, and 129, but a G in the consensus splice sequence and in Rs1 mice (B10.D2, B6). (B) RT-PCR analysis of Rslcan-9 liver RNAs. Diagrammed in $C$ is the Rslcan-9 gene with splice donor sites $\left(\mathrm{SS}_{1}\right.$ and $\left.\mathrm{SS}_{2}\right)$ identified in exon 1 and arrows indicating primers used for RT-PCR. Both transcripts are present in Rsl strains. The absence of the $\mathrm{SS}_{1}$ transcript in rsl strains is apparently the consequence of the IVS +5 mutation in $\mathrm{SS}_{1} \cdot(C)$ The $5^{\prime}$-ends of $\mathrm{SS}_{1}$ and $\mathrm{SS}_{2}$ transcripts are diagrammed. A hatched box represents a small open reading frame in the $\mathrm{SS}_{2}$ transcript that is out of frame with the KRAB-encoding exon and would likely interfere with translation from the second AUG. Both the first and second AUG codons are in frame in the $\mathrm{SS}_{1}$ transcript, suggesting this is the more translationally competent of the two. site (Fig. 5, $\mathrm{SS}_{1}$ ) encounters a second AUG 12 codons farther on in exon 2, encoding the beginning of the KRAB A domain. The methionine dictated by the second AUG is the second amino acid of the highly conserved KRAB A domain. Alternative cleavage at the second splice site, $\mathrm{SS}_{2}$, however, leads to the AUG in exon 2 being farther from and out of frame with the first, making translation of a functional KRAB-ZFP from this transcript unlikely (Fig. 5, SS ${ }_{2}$; Kozak 2002). In liver RNA of male and female Rs1 mice, both transcripts are detected by RT-PCR, but only $\mathrm{SS}_{2}$ is present in rsl mice. Thus, the IVS +5 mutation in rsl strains abolishes the $\mathrm{SS}_{1}$ transcript and likely compromises production of Rslcan-9 (Fig. 5C).

\section{$B A C$ transgenic rescue of the rsl phenotype}

To test functionality of these candidate genes, we asked whether wild-type BAC DNAs could rescue the mutant phenotype, that is, restore liver sexual dimorphism (Fig. 6). Adult $r s 1$ mice bearing BAC 163-B-15 transgenes (Fig. 3B) were screened for expression of MUPs, Slp, and Cyp2d9 (Fig. 6B, left). In a transgenic line carrying a partial BAC that appeared to be single copy and that only included Rslcan-13, Rslcan-4, and Rslcan-14, transgenic females expressed low levels of MUP protein and mRNA, but their nontransgenic sisters had high MUP levels characteristic of the rsl mutant phenotype. Female-specific Cyp2a4 levels were not affected, confirming rescue was specific to male-expressed genes. In another BAC 163-B-15 transgenic line, an internal deletion encompassed Rslcan-4; in these mice restoration of the Rsl phenotype did not occur (data not shown), supporting the hypothesis that Rslcan-4 was necessary for rescue. However, despite transgenic rescue of MUP sex-limitation, Slp and Cyp2d9 mRNAs were not reduced in mice homozygous for rsl (Fig. 6B; data not shown), suggesting that Rslcan-4 could regulate some but not all of the Rsl targets.

Rslcan-9, the paralog of Rslcan-4, was tested by transgenesis with BAC 45-N-22 (Fig. 3B) for the ability to regulate additional male-specific genes. As shown in Figure 6B (right panel), nontransgenic rsl females expressed Slp, but their transgenic sisters did not, indicating that SIp sex-limitation was restored. Liver sexual dimorphism in these transgenics was also enhanced for Cyp2d9, but was not apparent for MUP I/II. Furthermore, two additional transgenic lines with BAC 45-N-22 (Fig. 6C) makes apparent that reversion of the rsl phenotype correlates with the presence of the $\mathrm{SS}_{1}$ transcript for Rslcan-9 (see Fig. 5B).

Thus, as shown in the model of Figure 7 and discussed further below, Rslcan-4 and Rslcan-9 share regulation of sex-limitation, with each controlling a subset of the male-specific target genes. Inactivating mutations in each candidate lead to loss of sex-limitation, and wildtype genes can functionally restore liver dimorphism, confirming the identity of Rsl. Although it is possible that additional KRAB-ZFPs in these BACs participate in Rsl function, after extensive sequence comparison we have only found inactivating mutations in Rslcan-4 and 
Krebs et al.

A

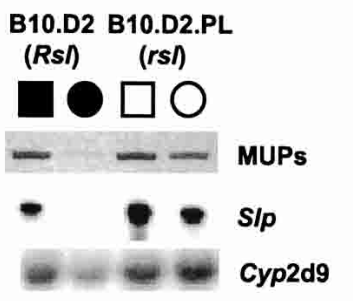

B

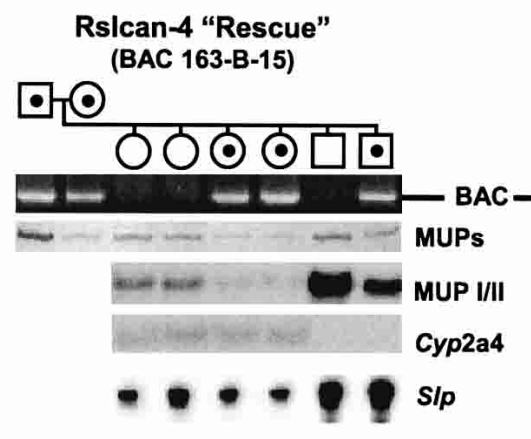

C

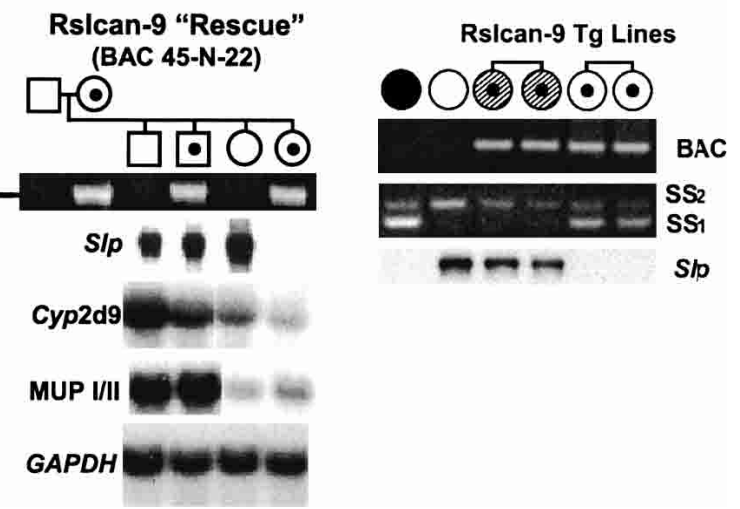

Figure 6. BAC transgenic rescue of the $r s$ phenotype. B6-derived BAC clones 163-B-15 and 45-N-22 were independently injected into oocytes, and transgenic mice were bred for homozygosity for PL/J markers flanking rsl. Transgenic mice (black dot in white circle) were identified by PCR of tail DNA for BAC sequences. To assess Rsl function, mouse urine was assayed for MUP protein by SDS-PAGE, liver mRNAs for MUP I/II and cyp2d9 by Northern blot, and SIp by RPA. GAPDH and cyp2a4 (female-prominent) were loading controls for Northern blots. C4 mRNA was an internal control for RPAs (data not shown). (A) Expression of MUP protein and Slp and cyp2d9 mRNA in inbred Rsl and $r s l^{P L}$ strains, for comparison. Expression of all target genes is elevated in rsl mice, most visibly in females. (B, left) For BAC 163-B-15 (containing B6-derived Rslcan-4), transgenic mice show reduced MUP protein in urine and MUP $I / I I$ mRNA in liver, but still have high Slp levels, relative to their nontransgenic siblings. This effect is more pronounced in females (e.g., cf. the two transgenic and the nontransgenic females). These results indicate that Rslcan-4 can restore dimorphic expression of MUP, but not Slp (lower panel) or cyp2d9 (data not shown). (Right) In contrast, mice transgenic for BAC 45-N-22 (containing B6-derived Rslcan-9) show reduced expression of Slp and cyp2d9, but expression of MUP I/II remains elevated (again, cf. the two females in the rightmost lanes). (C) Mice from two additional independent BAC 45-N-22 transgenic lines were assayed for liver expression of $R$ slcan-9 by RT-PCR, as in Figure 5. BAC DNA and Slp mRNA were assayed as above. For comparison, results from nontransgenic Rsl (filled circle) and rsl (open circle) females are at left. Representative sisters from one line (black dot in white circle) show expression of Rslcan-9 SS 1 transcript and extinguished SIp expression. In contrast, sisters of another line (black dot in hatched circle) only express $\mathrm{SS}_{2}$ and $\mathrm{Slp}$ remains elevated.

Rslcan-9. Even if other genes are involved, they must be functionally redundant. In any event, at least two genes, on two nonoverlappping BACs, are required to revert the rsl phenotype. The Human Genome Nomenclature Committee has assigned these genes the names Rsl1 (previously Rslcan-9) and Rsl2 (previously Rslcan-4). Rsl1 was so designated because it regulates Slp, which first revealed the existence of the Rsl pathway and which remains the hallmark of its action. Future studies will resolve the function of other genes in this region.

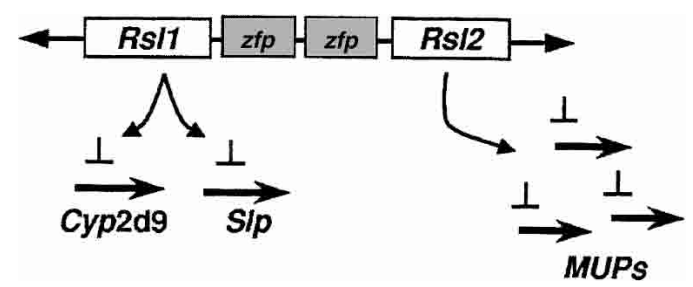

Figure 7. Rsl function is partitioned between two paralogous KRAB-ZFP genes. These findings indicate a division of labor between the recently duplicated Rsl1 (Rslcan-9) and Rsl2 (Rslcan-4), whereby one represses expression of MUP genes, and the other represses SIp and cyp2d9. This action sums to enforce sexually dimorphic expression of a set of genes that are hormonally induced in males at puberty. Biological functions of other ZFP genes in the region remain to be determined.

\section{Discussion}

Sexually dimorphic gene expression is extensive in rodent liver, where many of the affected proteins have roles in reproduction, either directly (via steroid metabolism, e.g. Cyp2d9) or indirectly (via pheromone communication, e.g., MUPs; Roy and Chatterjee 1983). Differential patterns of expression are established at puberty by gonadal steroids acting via the pituitary to elicit gender-dependent profiles of GH release (Lund et al. 1991). Variant rsl mice reveal that gene-specific repression, which is independent of androgen or GH influence, is as crucial to liver sexual dimorphism as is activation by hormonal cues: repression of male-specific genes in females greatly enhances the male-female difference (Tullis et al. 2003). The cloning of Rsl reveals the molecular basis of this repression, and further provides a biological model for a largely unstudied but huge class of regulators.

In accord with genetic and physiological evidence indicating that $R s l$ acts by negative regulation (Brown and Shreffler 1980; Tullis et al. 2003), positional cloning led to a chromosomal region replete with genes encoding KRAB zinc-finger repressor proteins (Fig. 3). There are nearly 200 of these genes in the mouse, many of which are arrayed in clusters (Ravasi et al. 2003). Their amplification has occurred relatively recently within the vertebrate lineage; whereas $\mathrm{C}_{2} \mathrm{H}_{2}$ zinc fingers are ancient, 
the KRAB domain only appears subsequent to the divergence of Fugu. The importance of these genes is suggested by their rapid proliferation, but their role in vivo is unclear because few natural target genes have been identified (Looman et al. 2002; Tanaka et al. 2002). Nevertheless, the mechanism of action of the KRAB-ZFPs has been well studied, in part because the KRAB domains encompass one of the strongest transcriptional repression modules characterized thus far (Thiel et al. 2001). The KRAB-ZFPs appear to function as monomers, and their sequence-specific DNA binding requires numerous contiguous zinc fingers (Peng et al. 2000; Gebelein and Urrutia 2001). The 28 -amino acid zinc-finger motif has highly conserved zinc-chelating Cys and His residues, but varies elsewhere, which likely determines distinct target gene recognition. The 75-amino acid KRAB domain mediates repression by recruiting an obligate and universal corepressor, KAP-1 (TIF1 $\beta$ ), that oligomerizes to form a scaffold coordinating both histone methylation and deposition of HP1 (heterochromatin protein 1; Friedman et al. 1996; Moosmann et al. 1996; Schultz et al. 2001). Thus, KRAB-ZFPs silence gene expression by nucleating local chromatin condensation. Given the detailed understanding of KRAB-ZFP action in general, it is expected that the Rsl genes function similarly, although this must be formally proven. Furthermore, although it is most likely that the malespecific liver genes are direct targets of Rsl repression, it is also conceivable that Rsl represses activators of these genes. Confirming the Rsl mechanism is a top priority subsequent to their identification. Perhaps most intriguing will be analysis of how hormonal induction and Rsl repression operate simultaneously in male mice.

A novel discovery resulting from the identification of Rsl is the sharing of a regulatory function by two paralogous genes (modeled in Fig. 7). Because Rs11 and Rsl2 (formerly Rslcan-9 and Rslcan-4) differ by less than a few percent over their entire sequence, having greater similarity to each other than any of their neighboring Rslcan genes, they likely result from a recent gene duplication event. Presumably a progenitor regulated the whole panel of male-specific genes, which were somehow partitioned between the daughters following their divergence. For example, modest differences in zinc-finger sequences could lead to preferences for some promoters over others. Although this preference seems qualitatively absolute based on results from the transgene rescue, where Rs11 regulates Slp and Cyp2d9, and Rsl2 controls MUPs, in actuality, the division of targets may be more quantitative and depend on the relative expression levels of Rs11 and Rs12. Thus, they may compete (or cooperate) differentially for the DNA regulatory binding sites, or for members of the repression machinery, such as KAP-1. Allelic differences in the targets, as well as in the Rsl genes, complicate comparisons between inbred strains and transgenic mice with mixed backgrounds. Division of target genes may be less than absolute and may underlie the quantitatively distinct phenotype of FM-derived mice, in which sex-limitation of MUPs as well as Slp is affected (Tullis et al. 2003). Whereas these mice carry the splice-site mutation in Rsl1, as yet there does not appear to be a mutation in Rsl2 (C.J. Krebs, L.K. Larkins, and D.M. Robins, unpubl.). Future experiments will determine relative expression levels of Rsl1 and Rsl2, as well as their relative affinities for distinct promoter sequences. This will help to discern to what extent the concerted action of the Rsl paralogs depends on the qualitative and/or quantitative regulation of their targets.

Because of previous detailed characterization of Rsl genetics and physiology (Brown and Shreffler 1980; Tullis et al. 2003), identification of these genes allows their immediate placement in a complex regulatory network. It is tempting to speculate that upstream regulators of Rsl, as well as additional downstream targets, may include other KRAB-ZFPs, perhaps from the same locus (Fig. 7). Furthermore, although this first assignment of KRAB-ZFP function pertains to liver physiology and sex differences, the numerous KRAB-ZFP genes in vertebrate genomes may intersect numerous biological pathways. It is interesting to note that several other gene families encoding transcriptional repressors, including domains such as BTB/POZ (Collins et al. 2001), seem also to have amplified recently in evolution. The general importance of transcriptional repression has been known since the classic analyses of prokaryotic systems, but recent evolutionary expansion of this regulatory mechanism implies its success in increasing diversity of higher organisms.

The comparative analysis of mouse and human draft sequences reveals that as many as $99 \%$ of mouse genes have an ortholog in man, underscoring the relevance of studying the function and regulation of these genes in mice (Mouse Genome Sequencing Consortium 2002). Intriguingly, the $1 \%$ of genes that do not have an exact human counterpart include differentially expanded members of multigene families that function in reproduction, xenobiotic metabolism, and immunity. It is reasonable to assume that differences conferred by these genes are involved in speciation events. Targets of Rsl (pheromone carriers, P450s, variant complement proteins) fall into the known classes of genes with members lacking orthologs in man. Rsl itself may also lack a human ortholog, because it maps to a break in synteny where expansion of the KRAB-ZFP genes likely postdates man-mouse divergence (Dehal et al. 2001). The human DNA sequences with synteny to either side of Rsl derive from Chromosomes 5 and 9, but the KRABZFPs with greatest similarity to Rsl are on human Chromosome 19. Further comparison will determine whether a functional ortholog of Rsl exists in the human genome. It is conceivable that differential expansion in gene families occurs not only for genes with functions related to speciation, but also within families encoding regulators of such genes.

Additionally intriguing from the evolutionary perspective is our observation of different mutations in both Rsl1 and Rsl2 genes in distinct inbred mouse lineages (Brown and Shreffler 1980; C.J. Krebs and D.M. Robins, unpubl.). Selection against $R s l$ in fact may be what al- 
lowed isolation of multiple "alleles" (haplotypes) of rsl mice. Because MUPS may influence pheromone-dependent aspects of puberty and fertility, their earlier and more abundant expression in rsl mice (Tullis et al. 2003) might result in earlier or more frequent litters, which would be of selective value in a laboratory setting. Therefore, discrete rsl haplotypes may have become artificially fixed for breeding vigor in the genomes of inbred mice. A prediction from this is that Rsl orthologs in human likely exist as pseudogenes, because reproduction in man, as in lab mice, may be less dependent on environmental constraints and chemical communication. Similar arguments apply to the silencing of pheromone receptor genes in the human genome (Giorgi et al. 2000; Rodriguez and Mombaerts 2002). Determining the function of Rsl may prove informative not just to liver physiology but also to aspects of reproduction and sexual maturation that are poorly understood, and for which experimental mouse models would be valuable.

In sum, by cloning Rsl we have elucidated the molecular mechanism of a regulatory axis that works in concert with hormonal induction to establish sexually dimorphic liver gene expression. Paralogous KRAB-ZFPs cooperate to repress transcription of male-specific liver genes. The implications of this first biological assignment of KRAB-ZFP function extend beyond liver physiology into cellular and behavioral aspects of reproduction. This, in turn, suggests a possible role for Rsl in speciation, and fuels speculation that other KRAB-ZFPs may be involved in similar evolutionary functions.

\section{Materials and methods}

\section{Mouse strains and genetic mapping}

B10.D2-Hc ${ }^{1}-\mathrm{H}_{2}{ }^{\mathrm{d}}-\mathrm{H} 2-\mathrm{T} 18^{\mathrm{c}} / \mathrm{nSnJ}$ (B10.D2; Jackson Labs \#000463), PL/J (Jackson Labs \#000680), 129S6/SvEvTac (129; Taconic Farms \#129SVEV), and C57BL/6J (B6; Jackson Labs \#000664) were obtained from the Jackson Laboratory and Taconic Farms. Congenic strains B10.D2.PL(1)-rsl (B10.D2.PL) and B10.D2.FM-rsl (B10.D2.FM) were originally obtained from Ray Miller (Jiang et al. 1996), and maintained at the University of Michigan. Homozygous B10.D2 (Rsl/Rsl) mice and homozygous $\mathrm{PL} / \mathrm{J}(r s 1 / r s l)$ mice were mated to produce an $\mathrm{F} 1$ generation, which was intercrossed to produce an F2 population. Recombinant mice were identified by PCR genotyping of Chromosome 13 markers from Research Genetics. All procedures involving mice were approved by the University of Michigan Committee on Use and Care of Animals, in accord with the NIH Guidelines for the Care and Use of Experimental Animals.

\section{RNA isolation and analysis}

Total RNA was isolated by the guanidinium isothiocyanate method (Ausubel et al. 1993). RNase protection assays and Northern blotting were performed as reported previously (Tullis et al. 2003). For RT-PCR, RNA was amplified with the Superscript One-Step RT-PCR kit (Invitrogen) according to the manufacturer's instructions. For radioactive RT-PCR, the forward primer was end-labeled with $\left[\gamma^{-32} \mathrm{P}\right] \mathrm{ATP}$ (ICN) and T4 polynucleotide kinase (New England Biolabs) according to instructions from NEB, prior to RT-PCR, and products were separated on $5 \%$ polyacrylamide/7 $\mathrm{M}$ urea sequencing gels followed by autoradiography.

\section{DNA isolation, subcloning, and analysis}

For PCR analysis, tail DNA was isolated according to Miller et al. (1988). For Southern blotting, liver DNA was isolated and 10 ug was restriction-digested, electrophoresed through agarose, and transferred to ZetaProbe GT Nylon Membranes (Bio-Rad) by standard procedures (Ausubel et al. 1993). PCR products for Rslcan-4 and Rslcan-9 from mouse strains B10.D2, B6, B10.D2.PL, 129, and B10.D2.FM were cloned into either pCR2.1-TOPO (Invitrogen) or pGEM-T-Easy (Promega), and were sequenced with $\mathrm{T} 7$ and Sp6 vector primers by the University of Michigan Sequencing Core Facility.

\section{Hybridization probes}

A mouse genomic DNA probe for Rslcan-4 was obtained by PCR using the following primers: 5 '-AAGGCCCTTAGTTCT CATAAAACAC-3' (forward), 5'-TCAGCCATGACAACATC CAT-3' (reverse). cDNA probes for MUP and Cyp genes were as reported previously (Tullis et al. 2003).

\section{Localization of genetic markers}

The genetic map was correlated with the physical map of the mouse genome using the Radiation Hybrid Mouse/Hamster Mapping Panel T31 from Research Genetics. PCR conditions were as recommended by the manufacturer, and results were obtained from the Jackson Laboratory Mouse Radiation Hybrid database by completing the form at their Web site (http://www. jax.org/resources/documents/cmdata/rhmap/rhsubmit.html).

\section{Identification of BAC clones}

A 443-bp portion of nonrepetitive B10.D2 genomic DNA adjacent to the D13Mit66 repeat was cloned using the Genome Walker kit from Clonetech and used to screen the C57BL/6J mouse BAC library RCPI-23 from Research Genetics, according to instructions provided. From this, 34 positive clones were identified, and their sequences were accessed from the NCBI database. The majority of the clones had end sequences in the database, and the entire sequences of BACs 45-N-22 and 163-B15 were entered as unordered fragments. Using similarity search programs such as BLAST (NCBI) and BAC End Sequence Database (TIGR), we found overlapping BACs that were also undergoing complete sequencing efforts. With this information and the Sequencher program v. 4.1 (GeneCodes), we assembled a virtual BAC contig consisting of six overlapping BAC clones (Fig. 3B).

To obtain clones from the RCPI-22 (129/SvEvTac) Research Genetics Library, a probe that hybridized to both Rslcan-4 and Rslcan-9 was sent to Research Genetics for custom screening. Address names for 45 positive BACs were provided, and 15 of those were purchased and typed by PCR for the presence of Rslcan-4, Rslcan-9, and adjacent polymorphic marker sequences. BACs 2-C-8 and 79-I-2 were among several that were positive for markers near Rslcan-4. BACs 404-K-24 and 104-N22 were positive for markers near Rslcan-9. To sequence BACs 404-K-24 and 79-I-2, plasmid libraries were made by SeqWright and screened by PCR; clones positive for Rsl candidate genes were sequenced end to end. 


\section{Analysis of BAC sequence}

Early attempts to identify genes within the BAC contig used the BLAST program to compare segments of the contig sequence with cDNAs in the NCBI database. This led to discovery of the previously unreported genomic sequence of mouse MZF22 and an intronless pseudogene encoding acidic ribosomal protein P0. Because MZF22 is a member of the KRAB zinc-finger gene family, whose members are putative transcriptional repressors and are often found in clusters in vertebrate genomes (Mark et al. 1999|, we used structural gene features such as exon order and proximity and intron/exon junctions to locate related genes within the contig. This strategy resulted in the identification of 22 additional KRAB zinc-finger genes (see Fig. 3).

\section{Transgenic animals}

To verify the functional existence of Rsl within the BAC clones, we rescued the rsl phenotype by microinjection of BAC DNA into fertilized oocytes, with the help of the University of Michigan Transgenic Animal Model Core. Owing to the unexpected inefficiency of fertilized egg production in vitro from a $\mathrm{PL} /$ $\mathrm{J} \times \mathrm{PL} / \mathrm{J}$ cross, BAC 163-B-15 transgenic founders were established in progeny from a female PL/J $(r s l / r s l) \times$ male B10.D2 $(R s 1 / R s 1)$ cross. To further maximize success in producing transgenic mice, BAC $45-\mathrm{N}-22$ was microinjected into $\mathrm{B} 6 \times \mathrm{SJL} / \mathrm{J} \mathrm{F} 1$ hybrids. Founder (FO) animals were backcrossed to $\mathrm{PL} / \mathrm{J}$ or B10.D2.PL mice and their progeny were typed by PCR for the transgene and for PL/J allele homozygosity at the Rsl locus. Mice 6 weeks of age were assayed for Rsl target gene expression, that is, MUP protein in urine $(1 \mu \mathrm{L}$ electrophoresed on $10 \%$ SDS-PAGE followed by Coomassie blue staining), and MUP, Slp, and Cyp2d9 mRNAs in liver after they were killed.

\section{GenBank accession numbers}

The GenBank accession numbers for the BAC contig (Fig. 3B) are as follows: 45-N-22 (\#AC079561), 349-F-18 (\#AC079534), 163-B-15 (\#AC079484), 408-H-6 (\#AC079547), 126-O-22 (\#AC079473), and 455-P-22 (\#AC079560). Accession numbers for Rslcan-9 (Rsl1), Rslcan-4 (Rs12), and Zfp455-460 (Rslcan-10, Rslcan-7, Rslcan-6, Rslcan-13, and Rslcan-14) are pending.

\section{Acknowledgments}

We thank our colleagues for helpful discussions throughout the course of this work, especially John Moran, Tom Glaser, David Kohrman, and members of the Robins lab past and present. We thank the excellent Cores at the University of Michigan, including those of the Michigan Diabetes Research and Training Center, the University of Michigan DNA Sequencing Facility, and the University of Michigan Transgenic Animal Models Core. This work was supported by a grant from the National Institutes of Health (D.M.R.).

The publication costs of this article were defrayed in part by payment of page charges. This article must therefore be hereby marked "advertisement" in accordance with 18 USC section 1734 solely to indicate this fact.

\section{References}

Ausubel, F.M., Brent, R., Kingston, R.E., Moore, D.D., Seidman, J.G., Smith, J.A., and Struhl, K. 1993. Current protocols in molecular biology. Greene Publishing Associates and WileyInterscience, New York.
Bacchini, A., Gaetani, E., and Cavaggioni, A. 1992. Pheromone binding proteins of the mouse Mus musculus. Experientia 48: 419-421.

Brown, L.J. and Shreffler, D.C. 1980. Female expression of the H-2 linked Sex-limited protein $(S I p)$ due to non-H-2 genes. Immunogenetics 10: 19-29.

Burge, C. and Karlin, S. 1997. Prediction of complete gene structures in human genomic DNA. J. Mol. Biol. 268: 78-94.

Collins, T., Stone, J.R., and Williams, A.J. 2001. All in the family: The BTB/POZ, KRAB, and SCAN domains. Mol. Cell. Biol. 21: 3609-3615.

Cox, B.J. and Robins, D.M. 1988. Tissue specific variation in C4 and Slp gene regulation. Nucleic Acids Res. 16: 6857-6870.

Danckwardt, S., Neu-Yilik, G., Thermann, R., Frede, U., Hentze, M.W., and Kulozik, A.E. 2002. Abnormally spliced $\beta$-globin mRNAs: A single point mutation generates transcripts sensitive and insensitive to nonsense-mediated mRNA decay. Blood 99: 1811-1816.

Dehal, P., Predki, P., Olsen, A.S., Kobayashi, A., Folta, P., Lucas, S., Land, M., Terry, A., Ecale Zhou, C.L., Rash, S., et al. 2001. Human chromosome 19 and related regions in mouse: Conservative and lineage-specific evolution. Science 293: 104 111.

Friedman, J.R., Fredericks, W.J., Jensen, D.E., Speicher, D.W., Huang, X.P., Neilson, E.G., and Rauscher III, F.J. 1996. KAP1 , a novel corepressor for the highly conserved KRAB repression domain. Genes \& Dev. 10: 2067-2078.

Gebelein, B. and Urrutia, R. 2001. Sequence-specific transcriptional repression by KS1, a multiple-zinc-finger-Kruppel-associated box protein. Mol. Cell. Biol. 21: 928-939.

Gebert, C.A., Park, S.H., and Waxman, D.J. 1997. Regulation of signal transducer and activator of transcription (STAT) 5b activation by the temporal pattern of growth hormone stimulation. Mol. Endocrinol. 11: 400-414.

Georgatsou, E., Bourgarel, P., and Meo, T. 1993. Male-specific expression of mouse Sex-limited protein requires growth hormone, not testosterone. Proc. Natl. Acad. Sci. 90: 3626 3630 .

Giorgi, D., Friedman, C., Trask, B.J., and Rouquier, S. 2000. Characterization of nonfunctional V1R-like pheromone receptor sequences in human. Genome Res. 10: 1979-1985.

Gustafsson, J.-A., Mode, A., Norstedt, G., and Skett, P. 1983. Sex steroid induced changes in hepatic enzymes. Ann. Rev. Physiol. 45: 51-60.

Jaffe, C.A., Ocampo-Lim, B., Guo, W., Krueger, K., Sugahara, I., DeMott-Friberg, R., Bermann, M., and Barkan, A.L. 1998. Regulatory mechanisms of growth hormone secretion are sexually dimorphic. J. Clin. Invest. 102: 153-164.

Jiang, P.P., Frederick, K., Hansen, T.H., and Miller, R.D. 1996. Localization of the mouse gene releasing sex-limited expression of Slp. Proc. Natl. Acad. Sci. 93: 913-917.

Kozak, M. 2002. Pushing the limits of the scanning mechanism for initiation of translation. Gene 299: 1-34.

Lakso, M., Masaki, R., Noshiro, M., and Negishi, M. 1991. Structures and characterization of sex-specific mouse cytochrome P-450 genes as members within a large family: Duplication boundary and evolution. Eur. J. Biochem. 195: 477486.

Looman, C., Abrink, M., Mark, C., and Hellman, L. 2002. KRAB zinc finger proteins: An analysis of the molecular mechanisms governing their increase in numbers and complexity during evolution. Mol. Biol. Evol. 19: 2118-2130.

Lund, J., Zaphiropoulos, P.G., Mode, A., Warner, M., and Gustafsson, J.-A. 1991. Hormonal regulation of cytochrome P450 gene expression. Adv. Pharmacol. 22: 325-354.

Margolin, J., Friedman, J., Meyer, W., Vissing, H., Thiesen, H.-J., 
Krebs et al.

and Rauscer III, F. 1994. Kruppel-associated boxes are potent transcriptional repression domains. Proc. Natl. Acad. Sci. 91: 4509-4513.

Mark, C., Abrink, M., and Hellman, L. 1999. Comparative analysis of KRAB zinc finger proteins in rodents and man: Evidence for several evolutionarily distinct subfamilies of KRAB zinc finger genes. DNA Cell Biol. 18: 381-396.

Miller, S.A., Dykes, D.D., and Polesky, H.F. 1988. A simple salting out procedure for extracting DNA from human nucleated cells. Nucleic Acids Res. 16: 1215.

Mode, A., Tollet, P., Strom, A., Legraverand, C., Liddle, C., and Gustafsson, J.-A. 1992. Growth hormone regulation of hepatic cytochrome $\mathrm{P} 450$ expression in the rat. Advan. Enzyme Regul. 32: 255-263.

Moosmann, P., Georgiev, O., Le Douarin, B., Bourquin, P., and Schaffner, W. 1996. Transcriptional repression by RING finger protein TIF1 $\beta$ that interacts with the KRAB repressor domain of KOX1. Nucleic Acids Res. 24: 4859-4867.

Mouse Genome Sequencing Consortium. 2002. Initial sequencing and comparative analysis of the mouse genome. Nature 420: $520-562$.

Nelson, S.A. and Robins, D.M. 1997. Two distinct mechanisms elicit androgen-dependent expression of the mouse Sex-limited protein gene. Mol. Endocrinol. 11: 460-469.

Osoegawa, K., Tateno, M., Woon, P.Y., Frengen, E., Mammoser, A.G., Catanese, J.J., Hayashizaki, Y., and de Jong, P.J. 2000. Bacterial artificial chromosome libraries for mouse sequencing and functional studies. Genome Res. 10: 116-128.

Peng, H., Begg, G.E., Harper, S.L., Friedman, J.R., Speicher, D.W., and Rauscher III, F.J. 2000. Biochemical analysis of the Kruppel-associated box (KRAB) transcriptional repression domain. J. Biol. Chem. 275: 18000-18010.

Ravasi, T., Huber, T., Zavolan, M., Forrest, A., Gaasterland, T., Grimmond, S., Members, R.G.G.a.G., and Hume, D.A. 2003. Systematic characterization of the zinc-finger-containing proteins in the mouse transcriptome. Genome Res. 13: $1430-1442$.

Rodriguez, I. and Mombaerts, P. 2002. Novel human vomeronasal receptor-like genes reveal species-specific families. Curr. Biol. 12: R409-R411.

Roy, A.K. and Chatterjee, B. 1983. Sexual dimorphism in the liver. Ann. Rev. Physiol. 45: 37-50.

Schultz, D.C., Friedman, J.R., and Rauscher III, F.J. 2001. Targeting histone deacetylase complexes via KRAB-zinc finger proteins: The PHD and bromodomains of KAP-1 form a cooperative unit that recruits a novel isoform of the Mi- $2 \alpha$ subunit of NuRD. Genes \& Dev. 15: 428-443.

Shahan, K., Gilmartin, M., and Derman, E. 1987. Nucleotide sequences of liver, lachrymal, and submaxillary gland mouse major urinary protein mRNAs: Mosaic structure and construction of panels of gene-specific synthetic oligonucleotide probes. Mol. Cell. Biol. 7: 1938-1946.

Shannon, M., Kim, J., Ashworth, L., Branscomb, E., and Stubbs, L. 1998. Tandem zinc-finger gene families in mammals: Insights and unanswered questions. DNA Seq. 8: 303-315.

Shreffler, D.C. 1982. MHC-linked complement components. In Histocompatibility antigens: Structure and function (Receptors and recognition, Series B) (eds. P. Parham and J. Strominger), pp. 187-219. Chapman and Hall, London.

Smith, J., Paton, I.R., Murray, F., Crooijmans, R., Groenen, M.A.M., and Burt, D.W. 2002. Comparative mapping of human Chromosome 19 with the chicken shows conserved synteny and gives an insight into chromosomal evolution. Mam. Genome 13: 310-315.

Symer, D.E., Connelly, C., Szak, S.T., Caputo, E.M., Cost, G.J., Parmigiani, G., and Boeke, J.D. 2002. Human L1 retrotrans- position is associated with genetic instability in vivo. Cell 110: $327-338$.

Tanaka, K., Tsumaki, N., Kozak, C.A., Matsumoto, Y., Nakatani, F., Iwamoto, Y., and Yamada, Y. 2002. A Kruppel-associated box-zinc finger protein, NT2, represses cell-type-specific promoter activity of the $\alpha 2$ (XI) collagen gene. Mol. Cell. Biol. 22: 4256-4267.

Tang, M., Waterman, M., and Yooseph, S. 2002. Zinc finger gene clusters and tandem gene duplication. J. Comput. Biol. 9: 429-446.

Thiel, G., Lietz, M., Bach, K., Guethlein, L., and Cibelli, G. 2001. Biological activity of mammalian transcriptional repressors. Biol. Chem. 382: 891-902.

Tullis, K., Krebs, C., Leung, J., and Robins, D. 2003. The Regulator of sex-limitation gene, Rsl, enforces male-specific liver gene expression by negative regulation. Endocrinology 144: $1854-1860$.

van den Berg, G., Veldhuis, J.D., Frolich, M., and Roelfsema, F. 1996. An amplitude-specific divergence in the pulsatile mode of growth hormone $(\mathrm{GH})$ secretion underlies the gender differences in mean GH concentrations in men and premenopausal women. J. Clin. Endocrinol. Metab. 81: 2460 2467.

Veldhuis, J.D. 1996. Gender differences in secretory activity of the human somatotropic (growth hormone) axis. Eur. J. Endocrinol. 134: 287-295.

Wassarman, D.A. and Steitz, J.A. 1992. Interactions of small nuclear RNAs with precursor messenger RNA during in vitro splicing. Nature 257: 1918-1925.

Waxman, D.J. 2000. Growth hormone pulse-activated STAT5 signalling: A unique regulatory mechanism governing sexual dimorphism of liver gene expression. Novartis Found. Symp. 227: 61-74.

Waxman, D.J., Ram, P.A., Pampori, N.A., and Shapiro, B.H. 1995. Growth hormone regulation of male-specific rat liver P450s 2A2 and 3A2: Induction by intermittent growth hormone pulses in male but not female rats rendered growth hormone deficient by neonatal monosodium glutamate. Mol. Pharmacol. 48: 790-797. 


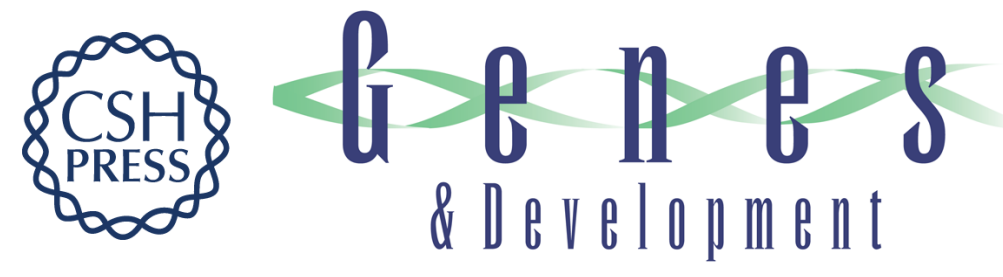

\section{Regulator of sex-limitation (RsI) encodes a pair of KRAB zinc-finger genes that control sexually dimorphic liver gene expression}

Christopher J. Krebs, Leslie K. Larkins, Ryan Price, et al.

Genes Dev. 2003, 17:

Access the most recent version at doi:10.1101/gad.1135703

References This article cites 43 articles, 17 of which can be accessed free at: http://genesdev.cshlp.org/content/17/21/2664.full.html\#ref-list-1

License

Email Alerting

Receive free email alerts when new articles cite this article - sign up in the box at the top Service right corner of the article or click here.

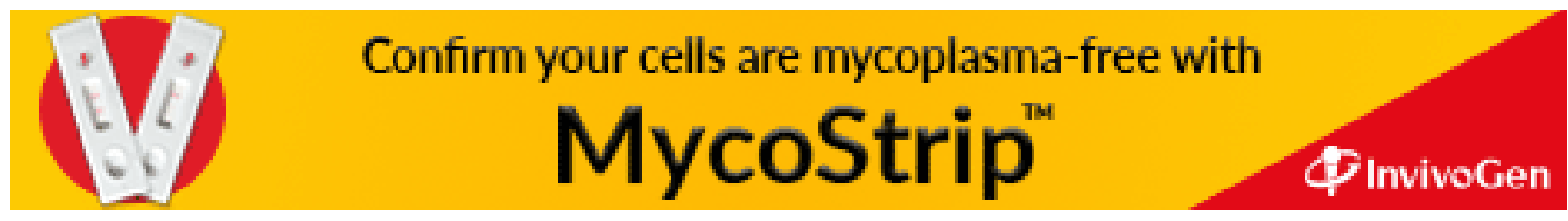

